\title{
SPICE circuit models for semiconductor lasers with effects of carrier and lattice heating
}

\author{
Chin-Yi Tsai ${ }^{a}$, Chih-Hsiung Chen ${ }^{a}$, Tien-Li Sung ${ }^{a}$, Chin-Yao Tsai ${ }^{b}$, and C. C. Yang ${ }^{c}$ \\ aDepartment of Electronic and Electrical Engineering, De Montfort University, Leicester LE1 9BH, \\ United Kingdom \\ ${ }^{b}$ Department of Electrical Engineering, National Cheng Kung University, Tainan, Taiwan \\ 'Department of Electrical Engineering and Graduate Institute of Electro-Optical Engineering, National \\ Taiwan University, Taipei, Taiwan
}

\begin{abstract}
A SPICE circuit model for semiconductor lasers is developed to simultaneously incorporate the effects of the carrier and lattice heating. Numerical simulations for the dc, ac and transient responses of this circuit model are demonstrated. The circuit model is transformed from the rate equations that govern the dynamics of carrier density, photon density, electron temperature, hole temperature and lattice temperature in the active region of semiconductor lasers. SPICE codes are developed exactly according to this circuit model. The results from this work demonstrate the capacities and versatilities of the SPICE circuits in simulating the complicated carrier and lattice heating processes for semiconductor lasers. The possibility of simulating optoelectronic systems in conjunction with electronic circuits and devices all by SPICE circuit models is also discussed.
\end{abstract}

Keywords: SPICE, circuit models, semiconductor lasers, carrier heating, lattice heating

\section{INTRODUCTION}

Developing circuit models for semiconductor lasers certainly has many advantages if they can then be used alongside other circuit models for design purposes. Computer-aided design (CAD) tools are gradually becoming indispensable for designing electronic processes, devices, circuits and systems, and in achieving the goal of electronic design automation (EDA) [1]. With thousands or even millions of transistors in a single chip, it is almost impossible to design electronic circuits or electronic systems using analytical methods without the help of CAD simulations. In a comparative manner, it is thus predictable that $\mathrm{CAD}$ tools for optoelectronic devices and systems will become critically important in the future. Through numerous efforts over more than three decades, CAD tools for electronics processes, devices, circuits and systems have been well developed. Several simulation packages have been commercialised, for example, SUPREM for processes, MEDICI and ATLAS for devices. Some of the simulation languages have become industrial standards, such as SPICE for analogue circuits [2], and VHDL or Verilog for digital circuits. Despite such success, it is surprising to find that the development of CAD tools for optoelectronic devices or systems is still not fully explored and as yet, no standard associated with them has emerged.

Generally, compared to their electronic counterparts, developing CAD tools for optoelectronic devices and systems is still in its infancy and currently lacks a commonly accepted standard. As a result, the approach to develop any simulation tool is either by establishing a new one or accommodating the well-established ones. For optoelectronic devices, especially optical transmitters, receivers converting electronic and optical signals, used in conjunction with other electronic devices, the latter seems a viable choice. Especially, for semiconductor lasers used in fibre optic communication systems as electronic driving circuits provide their electronic signals. As SPICE circuit simulation is widely used to design the driving circuits of semiconductor lasers, it is logical to develop SPICE circuit models for these devices. This has the advantage of using established software tools in an integrated systems approach. Unlike electronic devices that are usually characterised by current and voltage, while optoelectronic devices are normally characterised by light intensity and current. Since light 
intensity cannot be represented by any physical circuit quantities, modelling optoelectronic devices by SPICE circuit model is certainly not physically transparent and should be implemented with caution. Nevertheless, if the circuit model is well constructed to take account of the realistic physical models and sufficiently represents the physical properties of the optoelectronic device, it will certainly bring great benefit for designing the optoelectronic module working within an electronic system such as optical transmitter and receiver. This approach avoids modelling optoelectronic devices by standalone tools or languages. This provides the motivation for undertaking the work.

The importance of the theoretical models for the development of any semiconductor device is definitely beyond doubt. In fact, theoretical models for semiconductor devices have been well developed; and several popular commercial packages, such as MEDICI, ATLAS and DESSIS, have been widely used by industrial and academic institutions. Basically, any theoretical models for electronic devices should be able to describe the behaviour of electrons inside the devices. Theoretical models used for simulating the electronic devices can be categorised into three different methods based on the assumed nature of the carrier transport in the devices: the Monte Carlo analysis, partial differential equation (PDE) or balance equation, and ordinary differential equation (ODE) or rate equation. For each carrier, electron or hole, in a device, three parameters are needed to fully characterise its movement inside the device: time $(t)$, space (r) and momentum (k). The Boltzmann equation is used to describe the motions of all of the carriers inside the devices. Since it is almost impossible to analytically solve the Boltzmann equation even for a very simple and artificially defined case, a numerical method, such as the Monte Carlo method, is commonly implemented to solve the distribution of carriers in the $(t, \mathbf{r}, \mathbf{k})$ domain. Although, this method includes all the details of device physics, its simulation is rather complicated and time-consuming. In most cases, it does not provide a transparent explanation for the physical properties of the device.Alternatively, if the dynamics in the k-coordinate can be calculated by first principles or represented by average quantities (such as density, momentum and energy relaxation times), then the Boltzmann equations can be established by partial differential equations or balance equations. These balance equations can describe the carrier distribution (for example, density and temperature) in the temporal $(t)$ and spatial (r) domains. Since the numerical methods for solving PDE equations are highly developed, simulating the devices' properties by solving such balance equations are certainly tractable. This is the reason why such PDE balance equations are implemented in all the major simulation packages for electronic devices. However, since the measurable physical quantities such as current and voltage are only functions of time, this implies that all the spatial effects, such as carrier density and temperature distributions in the spatial domain, that affect the output current or voltage can be further simplified if they can be represented by some average quantities. They can totally be neglected if such spatial effects are insignificant. Averaging or neglecting the physical quantities in the spatial domain can further simplify the original PDE balance equations into the ODE rate equations. This dramatically simplifies the theoretical models and numerical procedures for device simulations. However, it should be noted that the approach is only valid if the spatial effect of carrier distribution can really be treated as an average or totally neglected.

Since carrier transport in semiconductor lasers is rather similar to other electronic devices, therefore all the theoretical models discussed for electronic devices are applicable to semiconductor lasers. In fact, the physical processes occurring in the active region, where electrons and holes recombine and generate photons, almost determining all the physical properties of semiconductor lasers. As a result, the spatial effects can usually be ignored (except, for example, the spatial-hole-burning effect in DFB lasers) and rate equations employed. This explains why rate equations are widely implemented for simulating the physical properties of semiconductor lasers. Although many commercial simulation packages use PDE balance equations, for example, PISC3D, the method of rate equations is the most popular theoretical approach indeed for modelling semiconductor lasers. Basically, all the major physical properties such as L-I relationship, linewidth, modulation response, chirp and noise can successfully be characterised by rate equations. Therefore, the rate-equation approach is used in this work. The SPICE circuit model proposed in this work is exactly achieved from the rate equations.

As discussed, it can be anticipated that developing SPICE models is invaluable for realising the EDA of optoelectronic devices, which are used in conjunction with electronic devices or systems. Following such a strong anticipation of future need, several SPICE models for semiconductor lasers have been proposed [3]-[14]. The circuit models for bulk semiconductor lasers, that is, heterostructure, derived from the carrier-photon rate equations were first proposed by Tucker [3][4]. Based on this model, Lu et al. developed a SPICE circuit model for quantum-well lasers by incorporating additional effects of carrier transport [5][6]. In contrast, instead of concentrating on the carrier transport effects which only became important in the high-speed modulation mode, the Xu group proposed SPICE circuit models for quantum well lasers particularly emphasising on the heating effects [7][8]. This is based on the fact that the nonlinearity in the L-I relationship of any semiconductor laser can satisfactorily be explained by considering the heating effect. Tsou et al. also proposed a SPICE circuit model for quantum-well lasers including the carrier-transport effect [9]. In addition, the effect of the coulomb enhancement on the gain coefficient and thus the properties of the high-speed modulation were investigated using their 
SPICE model [10]. A more evolved SPICE model including the carrier-transport effect was proposed by Rossi et al. [11]. They used their SPICE model to analyse and design $1.55 \mu \mathrm{m}$ quantum-well lasers for improving the high-speed performance. Madhan et al. proposed a SPICE circuit model for the multimode lasers and investigated the bistable behaviour of the devices [12]. Czotscher et al. produced an approach in which they combined their circuit model with the physical models of optical fibres and photodetectors to simulate the intensity modulation and chirp in a fibre optic communication system [13]. Recognising that heating has a paramount effect on the properties of vertical-cavity surfaceemitting lasers (VCSELs), Mena et al. proposed a SPICE circuit model with heating effects for VCSELs [14]. Mena et al. used their model to fit experimental data and thereby extracted the related physical parameters of the device. However, it should be noted that heating effects in their model and in the model of the $\mathrm{Xu}$ group are only referred to the lattice heating. Carrier heating effects are neither included nor discussed in their models.

From the foregoing it is seen that the temperature-dependent performance characteristics of devices are well established in SPICE circuit models. Consequently, physical quantities such as resistance and capacitance are assigned to a function of temperature (.TEMP) in the SPICE codes. Therefore, it is widely accepted that SPICE circuit models for semiconductor lasers should represent this temperature-dependent feature in order to further integrate their implementation into electronic circuits and increase their accuracy in predicting performance. In fact, the heating problem is well recognised as one of the most important factors influencing both the static and dynamic performance of semiconductor lasers. However, all the published SPICE models, which include heating effects in semiconductor lasers, solely refer to the lattice heating while the direct effect of the carrier heating has totally been neglected. It should be noted that the injection current supplies energy to carriers, which then release their energy to lattice via phonon emission. Since the injection current supplies energy directly to electrons and holes, not directly to lattice in a semiconductor laser, any issue regarding the lattice heating in semiconductor lasers will inevitably involve the issue of the carrier heating. Consequently, theoretical models used for predicting the heating effects on the performance of semiconductor lasers are thus anticipated to be able to simultaneously accommodate the physical mechanisms of the carrier and lattice heating. This certainly implies that any attempt to establish the temperature-dependent feature for SPICE circuit models of semiconductor lasers should be able to incorporate the features of the carrier and lattice heating in the same model, not just the lattice heating alone.

\section{RATE EQUATIONS}

It has been well recognised that the conventional carrier-photon rate equations can successfully describe many static and dynamic properties of semiconductor lasers. Therefore, any theoretical model that is proposed for further refinement should be able to accommodate these rate equations. Theoretical models for semiconductor lasers based on such a rate-equation approach certainly implies that the spatial effects of physical quantities will be either averaged or neglected. For example, the optical confinement factor is an average quantity characterising the overlap of the optical field with the carriers in the active region. The injection efficiency is an average quantity describing the percentage of current goes into the active region. The behaviours of the carrier and lattice heating outside the active region were neglected.

In order to incorporate the effects of the carrier and lattice heating, additional rate equations are needed to supplement the conventional carrier-photon rate equations. In this work, rate equations governing the carrier density $n$, photon density $s$, electron temperature $T_{e}$, hole temperature $T_{h}$ and lattice temperature $T_{L}$ will be used, that is, the five rate equations are used to describe the time variation of the carrier density $n$, photon density $s$, electron temperature $T_{e}$, hole temperature $T_{h}$

$$
\begin{aligned}
\frac{d n}{d t}= & \frac{\eta_{i m j} I_{i m j}-I_{\text {leak }}}{e V}-R_{S R H}-R_{s p o n}-R_{A u g}-v_{g} G s \\
\frac{d s}{d t}= & \Gamma v_{g} G s-\frac{s}{\tau_{s}}+\Gamma \beta R_{s p o n} \\
\frac{d T_{e}}{d t}= & \left(\frac{\partial u_{e}}{\partial T_{e}}\right)^{-1}\left[\left\langle\Delta E_{i m j}^{e}\right\rangle \frac{\eta_{i n j} I_{i m j}-I_{\text {leak }}}{e V}+\left\langle\Delta E_{s t i m}^{e}\right\rangle v_{g} G s+\left\langle\Delta E_{A u g}^{e}\right\rangle R_{A u g}^{e}\right. \\
& \left.+\left\langle\Delta E_{f c a}^{e}\right\rangle v_{g} \alpha_{f c a}^{e} s-\frac{u_{e}\left(T_{e}\right)-u_{e}\left(T_{L}\right)}{\tau_{e-L}}-\frac{u_{e}\left(T_{e}\right)-u_{e}\left(T_{h}\right)}{\tau_{e-h}}\right]
\end{aligned}
$$




$$
\begin{aligned}
\frac{d T_{h}}{d t} & =\left(\frac{\partial u_{h}}{\partial T_{h}}\right)^{-1}\left[\left\langle\Delta E_{i n j}^{h}\right\rangle \frac{\eta_{i n j} I_{i n j}-I_{\text {leak }}}{e V}+\left\langle\Delta E_{\text {stim }}^{h}\right\rangle v_{g} G s+\left\langle\Delta E_{\text {Aug }}^{h}\right\rangle R_{\text {Aug }}^{h}\right. \\
& \left.+\left\langle\Delta E_{f c a}^{h}\right\rangle v_{g} \alpha_{f c a}^{h} s-\frac{u_{h}\left(T_{h}\right)-u_{h}\left(T_{L}\right)}{\tau_{h-L}}+\frac{u_{h}\left(T_{h}\right)-u_{h}\left(T_{e}\right)}{\tau_{h-e}}\right] \\
\frac{d T_{L}}{d t} & =-\frac{T_{L}-T_{H S}}{\tau_{k}}+\frac{1}{c_{L} \rho_{L}}\left[\frac{u_{e}\left(T_{e}\right)-u_{e}\left(T_{L}\right)}{\tau_{e-L}}+\frac{u_{h}\left(T_{h}\right)-u_{h}\left(T_{L}\right)}{\tau_{h-L}}+\left(E_{F e}-E_{F h}\right) R_{S R H}\right]
\end{aligned}
$$

where $n$ only denotes the carrier density in the active region, $e$ is the unit of electron charge, $V$ is the volume of the active region, $I_{i n j}$ represents the external injection current, $\eta_{i n j}$ represents the percentage of the injection current that reaches the active region, $I_{\text {leak }}$ is the leakage current, $R_{S R H}, R_{s p o n}, R_{\text {Auger }}$ and $v_{g} G s$ represent the carrier recombination rates (i.e., loss rates) due to the Shockley-Read-Hall (SRH) recombination, spontaneous-emission recombination, Auger recombination and stimulated-emission recombination, $s$ is the photon density of the lasing light inside the cavity, $\Gamma, v_{g}, G$ and $\tau_{s}$ are confinement factor, group velocity, gain coefficient and photon lifetime, respectively, $\beta$ is the percentage of photons emitted by spontaneous emission coupling into the stimulated emission, $u_{c}(T), c=e$ or $h$, is the carrier energy density at temperature $T, \alpha_{f c a}^{c}$ is the coefficient of the free-carrier absorption, $\left\langle\Delta E_{\text {process }}^{c}\right\rangle$, process $=i n j$, stim, Aug and fca is the average energy change per carrier due to the processes of the injection, stimulated-recombination, Auger recombination and free-carrier-absorption heating, $T_{H S}$ is the temperature of the heat sink, $c_{L}$ is the specific heat capacity of the lattice, and $\rho_{L}$ is the material density of the lattice. In these rate equations, there are four time constants: $\tau_{\kappa}$ is the thermal conduction time, $\tau_{e-L}$ is the electron-lattice energy relaxation time, $\tau_{h-L}$ is the hole-lattice energy relaxation time and $\tau_{e-h}$ is the electron-hole energy relaxation time. These time constants govern the overall energy exchange rates between electrons, holes and lattice.

Apparently, (1) describes that the injection current supplies carriers into the active region, while the leakage, SRH, spontaneous emission, Auger and stimulated emission processes annihilate carriers in the active region. Similarly, (2) manifests that the stimulated emission and the coupling of the spontaneous emission supply the lasing photons, while the losses due to the internal loss and the mirror loss deplete the photons inside the cavity. From (3), it can be seen that the electron temperature will increase due to the injection heating, stimulated-recombination heating, Auger-recombination heating and free-carrier-absorption heating; and it will decrease due to the electrons releasing their energy to lattice and holes as they relax. In the same manner, as described in (4), the hole temperature will increase due to the same heating mechanisms and the receiving of electron energy. Similarly, it will decrease due to the holes releasing their energy to lattice. The lattice temperature will increase as it obtains energy from the electrons and holes via the intraband energy relaxation and the interband SRH process with multiple-phonon emission, as described in (5).

\section{SPICE CIRCUIT MODELS}

In order to implement the rate equations into the circuit models, the physical quantities described by the equations require to be transformed into circuit quantities which model each physical process as, for example, currents, voltages and passive elements. Perhaps the most challenging aspect in such a transformation is to adequately represent the carrier density by a circuit quantity or element.

The rate equation for carrier density (1) can be rewritten in a 'current form' as:

$$
\begin{aligned}
e V \frac{d n}{d t} & =\eta_{i n j} I_{\text {inj }}-I_{\text {leak }}-e V R_{S R H}-e V R_{\text {spon }}-e V R_{A u g}-e V v_{g} G s \\
& \equiv \eta_{i n j} I_{i n j}-I_{\text {leak }}-I_{S R H}-I_{\text {spon }}-I_{\text {Aug }}-I_{\text {stim }}
\end{aligned}
$$

where $I_{S R H}=e V R_{S R H}, I_{s p o n}=e V R_{s p o n}, I_{A u g}=e V R_{A u g}$, and $I_{s t i m}=e V v_{g} G s$ represent the recombination currents due to the SRH, spontaneous emission, Auger and stimulated emission, respectively. The task in transforming this rate equation is to choose a proper circuit element to represent the term $\mathrm{eV}(d n / d t)$. The most obvious one is by using a capacitor as: $e V(d n / d t) \rightarrow C_{n}\left(d V_{n} / d t\right) \propto I$. Since the value of the carrier density $n$ above the threshold is within the range of $10^{24} \mathrm{~m}^{-3}$, it is unreasonable to directly represent $n$ by any circuit element or value. To circumvent this problem, it is necessary to 
transform the carrier density $n$ into another physical quantity. Fortunately, the voltage drop $V_{n}$ in the active region of a semiconductor laser actually corresponds to the separation of the quasi-Fermi energies between electrons and holes, that is, $e V_{n}=E_{F e}-E_{F h}$. Therefore, since the carrier density is determined by its quasi-Fermi energy (i.e., $n\left(E_{F e}\right)$ and $n\left(E_{F h}\right)$ ), it is a function of $V_{n}$ as well; i.e., $n\left(V_{n}\right)$. As a result, if the change rate of the carrier density is implemented by a capacitor, then

$$
e V \frac{d n}{d t}=e V \frac{\partial n}{\partial V_{n}} \frac{d V_{n}}{d t} \equiv C_{n} \frac{d V_{n}}{d t}
$$

where the capacitor is defined by $C_{n}\left(V_{n}\right)=e V\left(\partial n / \partial V_{n}\right)$. It should be noted that the value of this capacitor strongly depends on $V_{n}$. In fact, it resembles the exponential relationship. In the SPICE syntax, the nonlinear element of a capacitor can only be represented by a second-order polynomial form as given in [1]. That is: $C(V)=C_{0}\left(1+a_{1} V+a_{2} V^{2}\right)$. Since the carrier density is exponentially dependent on the Fermi energy, it is almost impossible to represent $C_{n}\left(V_{n}\right)$ by such a second-order polynomial form with reasonable accuracy. However, such an approach has been used by the Xu group [7][8], Rossi et al. [11] and Mena et al. [14]. Unfortunately, no justification is given for representing the rate equation by $C_{n}\left(V_{n}\right)$ in their work, nor is the method by which the relationship is implemented the $C_{n}\left(V_{n}\right)$ in SPICE codes given. As a consequence of this discussion, it was decided not to use this approach in this work.

An alternative approach is to transform the change rate of the carrier density as: $e V(d n / d t) \rightarrow \tau_{n}\left(d I_{n} / d t\right) \propto I$. Such a transformation links the value of the carrier density to the value of the current. This can be achieved if the carrier density $n$ is transformed into a current $I_{n}$ by the definition: $I_{n} \equiv e V n / \tau_{n}$, where $\tau_{n}$ is arbitrary time constant. The reason for the choice is that since the value of $n$ is a function of $V_{n}$; the current $I_{n}$ should be a function of $V_{n}$. As a result, a circuit element must be found to represent the relationship between $I_{n}$ and $V_{n}$. Since the value of the carrier density $n$ is exponentially dependent on the value of the quasi-Fermi energy $E_{F c}$, and thus $V_{n}$, it can be expected that $I_{n}$ will also be exponentially dependent on $V_{n}$. Coincidently, the current in a diode exponentially depends on the voltage; therefore, $I_{n}\left(V_{n}\right)$ can be well represented by a diode. In SPICE, the $I-V$ relationship of a diode is described by

$$
I_{n}\left(V_{n}\right) \approx I_{s}\left[\exp \left(\frac{V_{n}-I_{n} R_{s}}{\eta_{d} V_{T}}\right)-1\right]
$$

where $V_{T}=k_{B} T / q, I_{s}$ is the saturation current, $R_{s}$ is the series resistor of the diode, and $\eta_{d}$ is the parameter to distinguish the different contribution between the diffusion current $\left(\eta_{d} \approx 1\right)$ and the recombination generation current $\left(\eta_{d} \approx 2\right)$. The parameters $I_{s}, R_{s}$ and $\eta_{d}$ are chosen to represent the real $I_{n}\left(V_{n}\right)$ as close as possible. In this model, $\eta_{d}=2$ is chosen to characterise the recombination feature in the active region. Note that at $V_{n}=0$ and $I_{n}=I_{s}$, the carrier density is the intrinsic carrier density for the intrinsic active region, i.e., $n=n_{i}$. Therefore, $I_{s}=e V n_{i} / \tau_{n}$ is used in this work. Representing $I_{n}=e V n\left(V_{n}\right) / \tau_{n}$ by a diode, (6) can be rewritten as

$$
\eta_{i n j} I_{i n j}=I_{\text {leak }}+I_{S R H}+I_{\text {spon }}+I_{\text {Aug }}+I_{\text {stim }}+\tau_{n} \frac{d I_{n}}{d t}
$$

Following this transformation, the physical meaning of the original rate equation is more transparent. For the dc case, that is, $\tau_{n} \cdot d I_{n} / d t=0$ in (9), the injection current is channelled into the leakage current, SRH recombination, spontaneous-emission recombination, Auger recombination or stimulated-emission recombination. Of course, the leakage, SRH and Auger process do not produce any light, so they are nonradiative recombinations. In contrast, the spontaneous-emission and stimulatedemission recombinations are radiative processes because they generate light: incoherent light by spontaneous emission and coherent light by stimulated emission.

After settling this controversial issue (representing the $d n / d t$ term), it appears almost impossible to directly represent $\tau_{n} \cdot d I_{n} / d t$ by a SPICE circuit. To circumvent this problem, a 'derivative circuit' with an artificial capacitor and a currentcontrolled voltage source $C_{n}\left(d V_{I_{n}} / d t\right)$ can be used to represent $\tau_{n}\left(d I_{n} / d t\right)$ utilising the following transformations: $\tau_{n} \rightarrow C_{n}$ and $I_{n} \rightarrow V_{I_{n}}$. Note that in the discussion, the arrow symbol is used to indicate the transformation of variables, although such a transformation may not preserve the physical unit of the original variables. For example, in this case, if $\tau_{n}$ 
$=10 \mathrm{~ns}$ is assumed, then its correspondent capacitance will be $C_{n}=10 \mathrm{nF}$. This part of circuit should be detached from the main circuits for carrier density. If it is connected to the main circuits for carrier density, then there will be a conflict between the voltage on the diode $V_{n}$ and the 'dummy' voltage $V_{I_{n}}$. However, because $V_{n}$ represents the quasi-Fermi energy separation in the active region, a physical quantity in which is interested, the diode is kept in the main circuit for carrier density and the circuit representing $C_{n}\left(d V_{I_{n}} / d t\right)$ is detached from the main circuit for carrier density. For this reason, $C_{n}\left(d V_{I_{n}} / d t\right)$ is termed a derivative circuit, because the main function of $C_{n}\left(d V_{I_{n}} / d t\right)$ is to represent $\tau_{n}\left(d I_{n} / d t\right)$ in the circuits.

The next step taken is to transform the photon rate equation into a circuit equation bearing in mind that $I_{\text {spon }}=e V R_{\text {spon }}$ and $I_{\text {stim }}=e V v_{g} G s$ in the device are to represent the real spontaneous and stimulated recombination currents in the circuit model. Multiplying (2) on both sides by $\mathrm{eV} / \Gamma$, this gives

$$
I_{\text {stim }}+\beta I_{\text {spon }}=\frac{e V}{\Gamma} \cdot \frac{s}{\tau_{s}}+\frac{e V}{\Gamma} \cdot \frac{d s}{d t}
$$

Since the unit on the right-hand side of (10) is current, and only the circuit element of a capacitor has the characteristics that the time derivative of its voltage equals its current, that is, $C_{s}\left(d V_{s} / d t\right)=I_{s}$; there is no choice but to relate the photon density to the voltage, that is, $s \propto V_{s}$. In addition, if the photon density is chosen to be related to the current, $s \propto I_{s}$, then $(\mathrm{eV} / \Gamma) \cdot(d s / d t)$ will be in the form of $\tau_{s}\left(d I_{s} / d t\right)$. The drawback of this latter choice is that another additional "derivative circuit" becomes involved, which is more complicated than incorporating a simple capacitor. Therefore, a voltage in this work will represent the photon density. However, it can be proved to be very impractical if the photon density $s$ is directly transformed into voltage, since usually $s \approx 10^{20} \sim 10^{22} \mathrm{~m}^{-3}$ when the device is biased above the threshold. In addition, the SPICE complier will not accept such a range of voltage values. Fortunately, the optical power $P$, which directly relates to the photon density $s$, emitted by a laser diode is usually within the $\mathrm{mW}$ range. This suggests that the emitted optical power turns out to be a suitable variable for transforming photon density $s$ into the voltage unit having a $\mathrm{mV}$ range. It is well known that the total output power from two mirror facets is related to the photon density $s$ by:

$$
P=\frac{\hbar \omega_{l} v_{g} \alpha_{\text {mirr }} V}{\Gamma} s
$$

Defining the 'optical voltage' by $P[\mathrm{~W}] \rightarrow V_{s}[\mathrm{~V}]$, the final circuit equation for the photon rate equation becomes:

$$
I_{\text {stim }}+\beta I_{\text {spon }}=\frac{V_{s}}{R_{s}}+C_{s} \frac{d V_{s}}{d t}
$$

where the resistor $R_{s}$ and the capacitor $C_{s}$ are defined by $\hbar \omega_{l} \tau_{s} / e \tau_{m} \rightarrow R_{s}$ and $e \tau_{m} / \hbar \omega_{l} \rightarrow C_{s}$, respectively, and the mirror lifetime of a photon $\tau_{m} \equiv 1 / v_{g} \alpha_{\text {mirr }}$ is used in the equations.

Transforming the rate equation for the electron temperature into a circuit model follows a similar pattern. First, some coefficients are defined as the following:

$$
\begin{aligned}
& \gamma_{i m j}^{e} \equiv \frac{\tau_{e-L}}{e V}\left(\frac{\partial u_{e}}{\partial T_{e}}\right)^{-1}\left\langle\Delta E_{i n j}^{e}\right\rangle, \gamma_{s t i m}^{e} \equiv \frac{\tau_{e-L}}{e V}\left(\frac{\partial u_{e}}{\partial T_{e}}\right)^{-1}\left\langle\Delta E_{s t i m}^{e}\right\rangle, \\
& \gamma_{\text {Aug }}^{e} \equiv \frac{\tau_{e-L}}{e V}\left(\frac{\partial u_{e}}{\partial T_{e}}\right)^{-1}\left\langle\Delta E_{\text {Aug }}^{e}\right\rangle, \gamma_{f c a}^{e} \equiv \frac{\tau_{e-L}}{e V}\left(\frac{\partial u_{e}}{\partial T_{e}}\right)^{-1}\left\langle\Delta E_{f c a}^{e}\right\rangle,
\end{aligned}
$$

and the free-carrier-absorption current is represented by $I_{f c a}^{e} \equiv e V v_{g} \alpha_{f c a s}^{e}$. Note that $u\left(T_{e}\right) \cong\left(\partial u_{e} / \partial T_{e}\right) T_{e}$ can be used and also it can be assumed that $\left(\partial u_{e} / \partial T_{e}\right) \approx\left(\partial u_{h} / \partial T_{h}\right)$. Since $u_{e} \approx 3 n_{e} k_{B} T_{e} / 2, u_{h} \approx 3 n_{h} k_{B} T_{h} / 2$ and $n_{e}=n_{h}$ are also assumed in this work, this assumption implies that $T_{e} \approx T_{h}$. As long as the electron temperature is not significantly different from the hole temperature, this assumption seems to be reasonable. Implementing the assumption, (3) becomes

$$
\gamma_{\text {inj }}^{e}\left(\eta_{i n j} I_{\text {inj }}-I_{\text {leak }}\right)+\gamma_{\text {stim }}^{e} I_{\text {siim }}+\gamma_{\text {Aug }}^{e} I_{\text {Aug }}^{e}+\gamma_{f c a}^{e} I_{f c a}^{e}=\left(T_{e}-T_{L}\right)+\frac{\tau_{e-L}}{\tau_{e-h}}\left(T_{e}-T_{h}\right)+\tau_{e-L} \frac{d T_{e}}{d t}
$$


Since the physical quantities on the left-hand side of (13) are currents, the same unit on the right-hand side of (14) should be kept as well. Therefore, either $C_{e}\left(d V_{e} / d t\right)$ or $\tau_{e}\left(d I_{e} / d t\right)$ can be used to represent the $\tau_{c-l}\left(d T_{c} / d t\right)$ term on the right-hand side of (14) to match the unit of current. However, since $C_{e}\left(d V_{c} / d t\right)$ only involves a single capacitor, while $\tau_{c}\left(d I_{e} / d t\right)$ needs an additional 'derivative circuit'. $C_{e}\left(d V_{c} / d t\right)$ is chosen rather than $\tau_{c}\left(d I_{c} / d t\right)$ to represent $\tau_{c-L}\left(d T_{e} / d t\right)$. Now since the temperatures $T_{e}, T_{h}$ and $T_{L}$ in the device are usually of the order of $300 \mathrm{~K}$, it is sensible directly to represent them by voltages: $T_{c}[\mathrm{~K}] \rightarrow V_{c}[\mathrm{~V}], T_{h}[\mathrm{~K}] \rightarrow V_{h}[\mathrm{~V}], T_{l}[\mathrm{~K}] \rightarrow V_{L}[\mathrm{~V}]$, and the capacitor by $\tau_{e l}[\mathrm{~s}] \rightarrow C_{c}[\mathrm{~F}]$. It should be noted that the values of voltage, resistance and capacitance defined in this part of the circuits do not really represent the physical units of volts, ohms and farads. They represent the values of the original variables instead. For example, the voltage $I_{1}$, in volt actually represents the lattice temperature $T_{L}$ in Kelvin. The original physical unit of $\gamma$ is $\mathrm{K} / \mathrm{A}$ while it is dimensionless in (14).
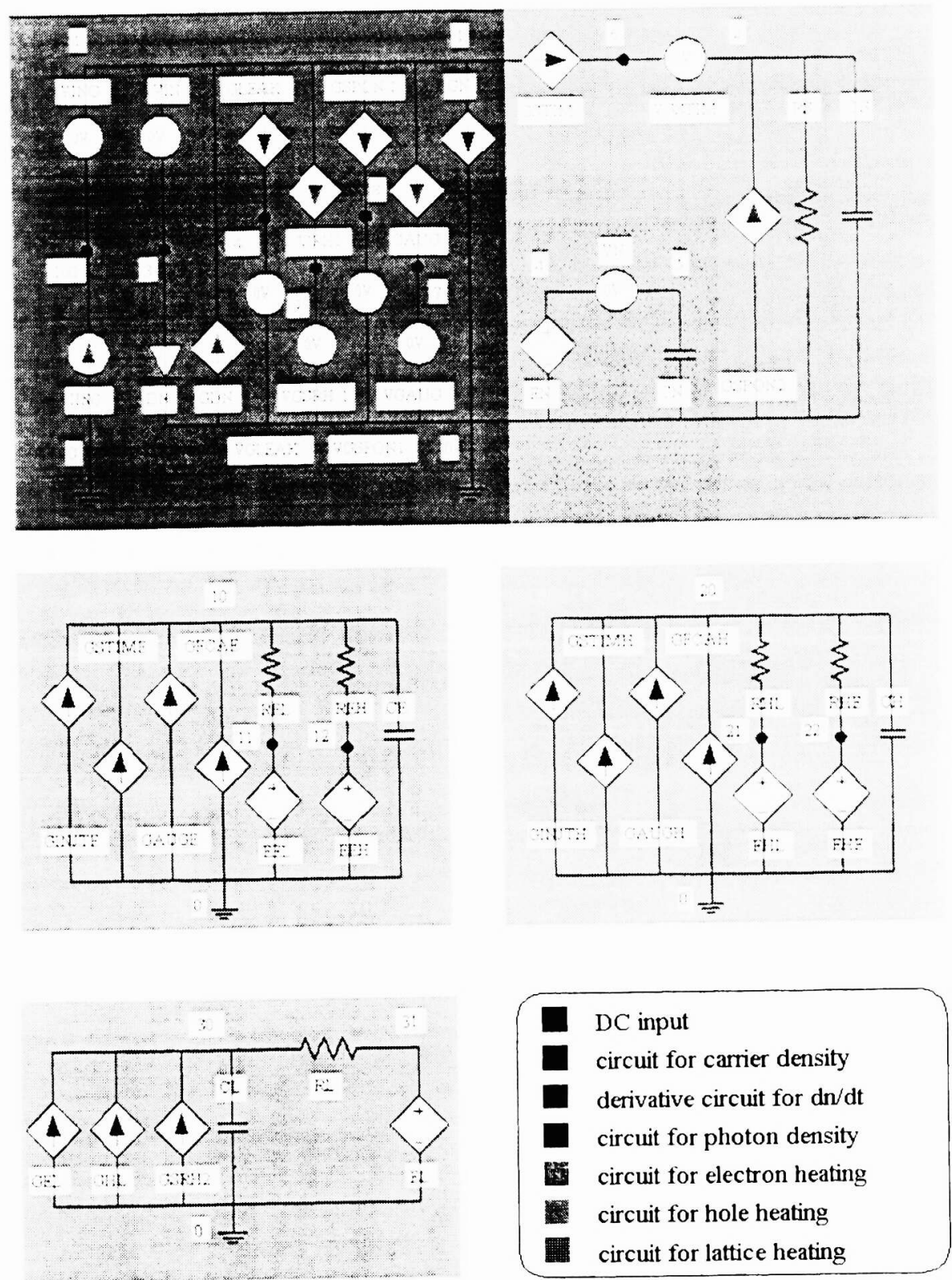

Fig. 1. SPICE circuit model with carrier and lattice heating. 
The choice of representing the electron temperature $T_{e}$ by a voltage $V_{e}$ certainly causes difficulties for the first and second term on the right-hand side of (14) because of the disagreement among their units with others. To circumvent this problem, two 'dummy' resistors are used $1 \Omega \rightarrow R_{e L}$ and $\tau_{e-h} / \tau_{e-L}[] \rightarrow R_{e h}[\Omega]$ adding to the first and second terms in (14), respectively. As a result, the units of all the terms in (14) are currents. Finally, the rate equation of the electron temperature becomes

$$
\gamma_{\text {inj }}^{e}\left(\eta_{\text {inj }} I_{\text {inj }}-I_{\text {leak }}\right)+\gamma_{\text {stim }}^{e} I_{\text {stim }}+\gamma_{\text {Aug }}^{e} I_{\text {Aug }}^{e}+\gamma_{f c a}^{e} I_{f c a}^{e}=\frac{V_{e}-V_{L}}{R_{e L}}+\frac{V_{e}-V_{h}}{R_{e h}}+C_{e} \frac{d V_{e}}{d t}
$$

which can be implemented into SPICE codes. By a similar derivation, transforming the rate equation for the hole temperature into a circuit model can be achieved by setting the hole temperature $T_{h}$ to a voltage $V_{h}$ and $T_{L}$ to a voltage $V_{L}$. The circuit model based on the results of (9), (11), and (140 is shown in Fig. 1.

\section{RESULTS AND DISCUSSION}

Once the circuit models are given, it will be rather straightforward to write a SPICE code according to the circuit models. Though, attentions should be paid to the numerical limitations of the SPICE complier itself. SPICE provides three basic features to probe and characterise a circuit system: dc, ac and transient responses. Generally, these three features are also used to characterise the properties of a semiconductor laser. Of course, the ac response and the transient response characterise the same temporal behaviour of the device. The ac response represents the system properties in the frequency domain while the transient response directly characterises the system behaviour in the time domain. For semiconductor lasers, the ac response is generally used to characterise the bandwidth of the devices. In addition, the transient response is usually employed to describe the turn-on behaviour of the devices. These built-in features in simulating the dc, ac and transient responses unquestionably give SPICE circuit models an unambiguous merit over their counterpart, the rate equations. For example, in order to simulate the ac response, a small-signal analysis on the rate equations must be provided. Such an analysis can be rather involved (sometimes unnecessarily complicated) especially in the case when dealing with the issues of the carrier and lattice heating. In addition, different numerical procedures must be implemented separately for the ac and transient responses when using the rate-equation approach. This certainly adds more numerical complexity onto the theoretical models themselves, even to the models representing the same device. Apparently, the simplicity and consistency in simulating the dc, ac and transient responses by using the SPICE circuit model provides a definite merit to allow the extraction of the physical parameters from the measurement data of the device. It should be kept in mind that, the purpose of the simulations presented in this work is not to exhaustively evaluate the effect of each parameter on the performance of the device, or to extract the values of these parameters by fitting the simulation results with the measurement data. The simulation presented in the following is for the purpose of demonstrating the capabilities especially with respect to the influences or performance of the heating effects and versatilities of the SPICE circuit models for semiconductor lasers.

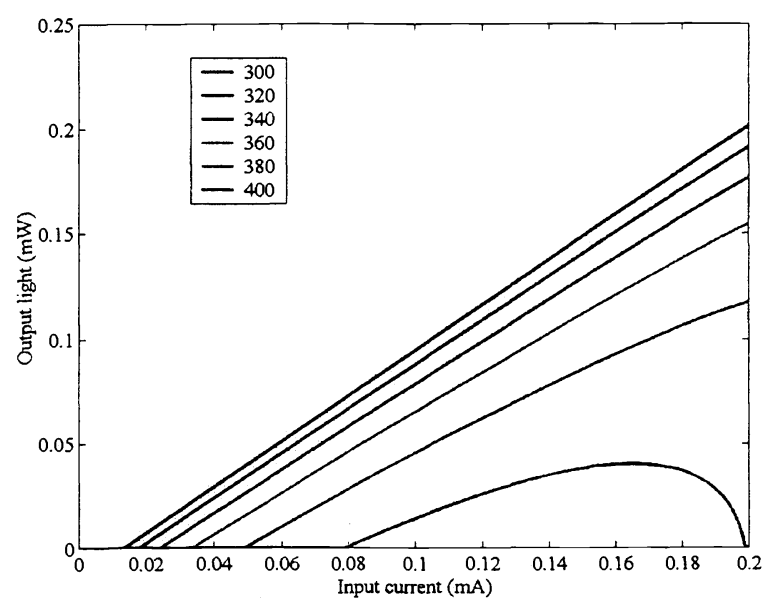

Fig. 2. L-I relationship of a semiconductor laser simulated with the temperature-dependent feature of the leakage current and Auger process for different heat-sink temperatures $T_{H S}$ (in the unit of Kelvin). 
In practice, the heating effects are usually investigated by changing the heat-sink temperature. This is because the changes of the carrier or lattice temperature within the device due to the injection current is almost impossible to directly measure by experimental means, and can only be estimated by theoretical tests. Of course, this work is useful in assessing the carrier and lattice temperatures in the active region of the devices but is carried out following device fabrication. However the circuit models developed can be used to simulate realistic experimental situations by changing the heat-sink temperatures $T_{H S}$ in the SPICE codes. The simulation results of the L-I relationship for different heat-sink temperatures $T_{H S}$ are shown in Fig. 2. Apparently, increasing the heat-sink temperature increases the threshold current and L-I nonlinearity. In this simulation, if the heat-sink temperature changes from $300 \mathrm{~K}$ to $400 \mathrm{~K}$, the threshold current will change from $10 \mathrm{~mA}$ to 80 $\mathrm{mA}$. This certainly validates that the threshold current is almost exponentially dependent on the heat-sink temperature: $I_{t h} \propto \exp \left(T_{H S} / T_{0}\right)$, where the characteristic temperature $T_{0} \approx 50 \mathrm{~K}$ is extracted from the simulation.

The L-I nonlinearity is usually attributed to the lattice heating if the heat-sink temperature is kept at a constant value. However, the results in the simulation shown in Fig. 2 demonstrate that the L-I nonlinearity can be influenced by the electron energy relaxation time $\tau_{e-L}$. Of course, attributing all heating effects just to the lattice heating is not only physically unrealistic but also numerically questionable. Incorporating the carrier heating into the heating model, which appears necessary and indispensable if realistic physical models are to be established.

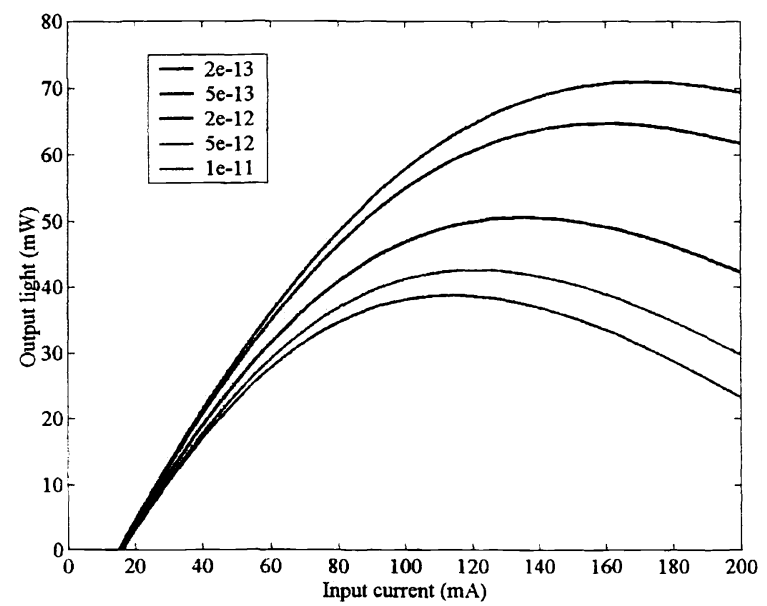

Fig. 3. L-I relationship for different electron-lattice energy relaxation times $\tau_{e-L}$ (in the unit of second).

The transient responses simulated by SPICE for the output power and electron temperature with different electron-lattice energy relaxation time $\tau_{e-L}$ are shown in Fig. 4 and 5, respectively.

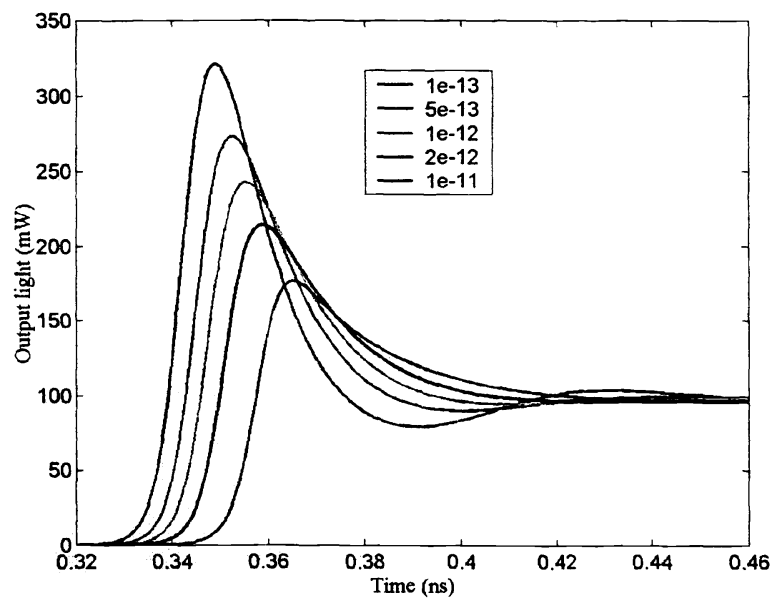

Fig. 4. Transient response of the output light for different electron-lattice energy relaxation times $\tau_{e-L}$ (in the unit of second). 
Unambiguously, the results in Fig. 4 show that increasing $\tau_{e-L}$ will increase the damping rate $\gamma$ in the transient response case. That is, the carrier heating will result in a damping in the dynamic properties of a semiconductor laser. The electron and hole temperatures will rapidly increase as the injection current switches on at $t=0 \mathrm{sec}$. Initially, only a small number of carriers exist in the active region, and the sudden supply of the injection heating will be distributed among them. As a result, each carrier will gain a huge amount of energy and thus the carrier temperature will suddenly rise to a dramatic level. As the heated carriers start to release their energy and the carrier density gradually increases as well, the carrier temperature will then progressively cool down. As the laser light starts to build up inside the cavity and then emitted by the mirrors, the stimulated recombination and free-carrier-absorption heating effects will heat carriers. Consequently, the carrier temperature will raise again, the increase of the carrier temperature being proportional to the photon density. After the initial transient variation the photon density settles down to its dc value, that is, the steady-state, and the carrier temperature will correspondingly settle down to its dc value. It should be noted that the small increase of the electron temperature between $0.1 \mathrm{~ns}$ and $0.2 \mathrm{~ns}$ in Fig. 5 is resulted from the Auger heating that will becomes effective when the carrier density in the active region reaches a certain value.

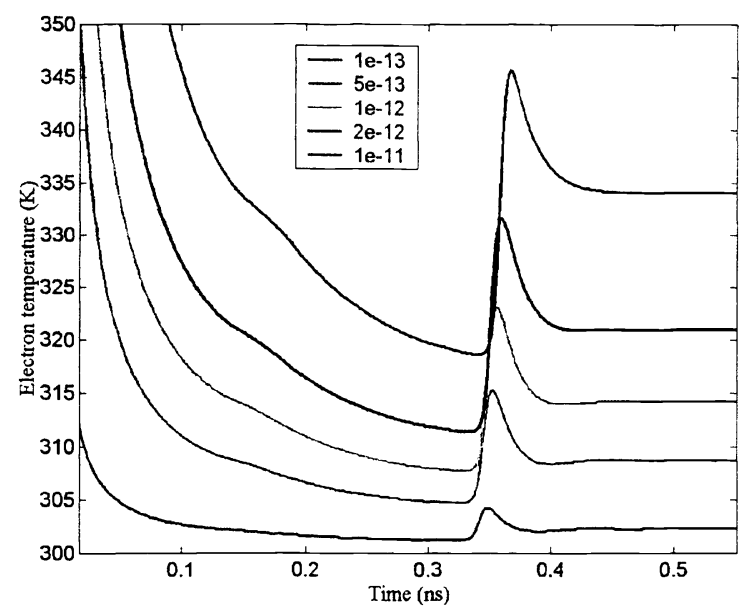

Fig. 5. Transient response of the electron temperature for different electron-lattice energy relaxation times $\tau_{e-L}$ (in the unit of second).

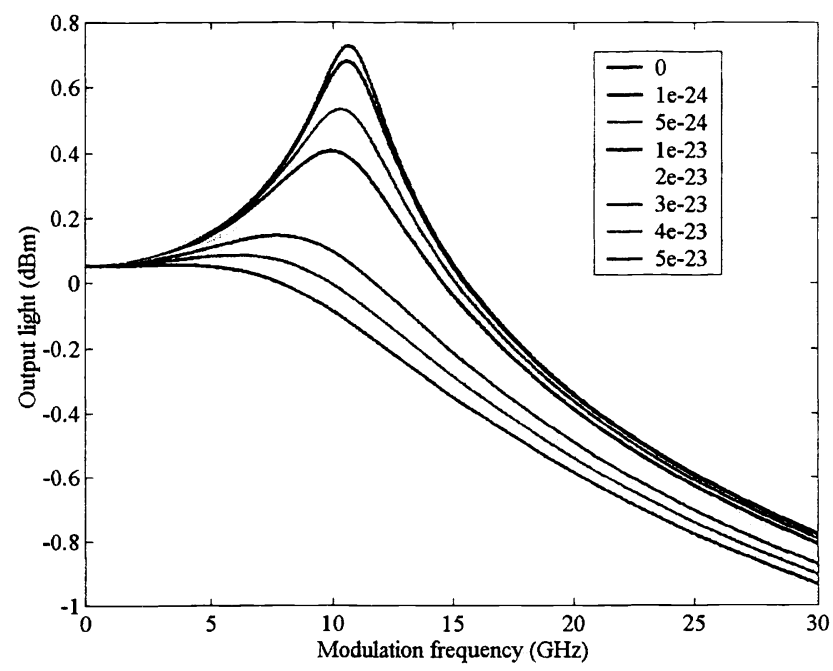

Fig. 6. AC response of a semiconductor laser with different nonlinear gain coefficients $\varepsilon_{\text {shb }}$ (in the unit of $\mathrm{m}^{3}$ ). 
The damping caused by the carrier heating coincidentally has similar features to the damping due to the spectral-hole burning. As a result, the effects of the carrier heating are conventionally included into the nonlinear gain coefficient $\varepsilon_{\text {shb }}$ as the effect of spectral-hole burning. The value of the nonlinear gain coefficient $\varepsilon_{\text {shb }}$ only affects the damping rate and does not significantly affect the value of the resonant frequency. This is also clearly verified by the simulation results shown in Fig. 6.

\section{CONCLUSION}

In this thesis, a new proposed SPICE circuit model for semiconductor lasers incorporating the effects of the carrier and lattice heating, which has been developed, implemented and realised. The circuit is achieved from the rate equations that govern the dynamics of carrier density, photon density, electron temperature, hole temperature and lattice temperature in the active region of semiconductor lasers. The set of the rate equations used provide a solid, sound and physically based theory for quantitatively describing the carrier and lattice heating in semiconductor lasers without unnecessary non-physical arguments (such as $I \cdot V-P$ in the previous work) to justify the heating effects. Based on this physical model, the rate equations have been transformed into a circuit model by tactically choosing suitable circuit elements and quantities to represent the physical quantities in the rate equations. All the physical quantities that depend on the carrier temperature (such as gain, the Auger process and leakage current) are explicitly included in the circuit model by representing them as voltage-controlled current source, the carrier temperatures then being represented by voltages. SPICE codes have been developed exactly according to this circuit model. It has been shown and demonstrated that this single SPICE circuit model alone can simulate the dc, ac (i.e., small signal in the frequency domain), and transient (i.e., large signal in the time domain) responses of semiconductor lasers. The new proposed SPICE circuit thus fully meets the initial objectives set, i.e. it is universal, physically based, flexible and expandable.

Without doubt, the role of Electronic Design Automation (EDA) in the modern electronic industries cannot be over emphasised. SPICE circuit simulation is almost indispensable in designing analogue electronic circuits. The needs of the SPICE circuit models for semiconductor lasers used in conjunction with other electronic circuits and systems (for example, the transmitter modules of fibre optic communication systems) are thus foreseeable. One of the major merits of using SPICE circuit models for devices is its flexibility in modifying the developed SPICE codes to accommodate more physical properties associated with devices. In other words, any physical properties that can be transformed into the models of resistances, capacitances, inductance, voltage and current sources can be incorporated into the original circuits without too many elaborate efforts. This suggests that further work can be under taken in order to improve the new SPICE circuit models presented in this work, for example:

1) In this work, the new SPICE circuit model only simulated the physical processes in the active region. In other words, the physical processes outside the active region were either neglected (for example, the heating mechanism outside the active region) or averaged (such as the injection efficiency). It should be noted that, in vertical-cavity surface-emitting lasers (VCSELs), the heating processes in the distributed Bragg reflection (DBR) mirrors could be as significant as in the active region. Under such a circumstance, additional circuits are needed to correctly model the overall heating behaviour within the devices. To incorporate these physical processes into the circuit model is certainly feasible; bui attentions should be paid to the numerical complexity or numerical stability for SPICE codes with too many circuit elements.

2) In this work, only the intrinsic physical properties were considered for semiconductor lasers. In other words, their extrinsic properties, such as parasitic resistances and capacitances associated with the devices themselves or the external wire bonding, were not considered. In practice, the parasitic properties might be an important factor affecting the properties of semiconductor lasers, especially in their high-speed performance. Including parasitic resistances and capacitances into the SPICE circuit model will be reasonably straightforward. However, their values usually cannot directly be assessed by either experimental measurements or theoretical calculations. But, if the parasitic resistances and capacitances are included into the circuit model presented in this work, their values can be estimated by measured ac data. This feature demonstrates another merit of using an extensive SPICE circuit model in characterising the properties of semiconductor lasers.

3) Only bulk semiconductor lasers are used as the numerical examples in this work, while quantum-well (QW) lasers are not particularly discussed. However, the physical models presented in this work are general and can be applied to QW lasers if the correspondent parameters are given. The carrier and lattice heating processes in QW lasers should be rather similar to those in bulk lasers. It has been shown that the carrier energy relaxation time for QW lasers calculated by first principles do not present any considerable difference from those for bulk lasers. On the other hand, the differential gain of QW lasers is substantially higher than that of bulk lasers. In addition, it should be noted that, other physical processes, such as the carrier transport, capture and escape, are not presented in bulk lasers but unique to QW lasers. In 
some cases, for example, if the separate confinement heterostructure ( $\mathrm{SCH})$ is very large, their effects may become crucial. Under such a circumstance, additional rate equations are needed to characterise their effects. Accordingly, extra circuits should be incorporated to describe the carrier transport, capture and escape processes.

4) The SPICE circuit model provides an indisputable advantage for designing semiconductor lasers used in conjunction with other electronic circuits or systems, especially in designing the transmitter modules for fibre optic communication systems. The transmitter modules, receiver modules and the optical fibres are the three major components in a fibre optic communication system. It is thus perceivable that, if SPICE circuit models can be further implemented for the receiver modules and optical fibres, they can be used to simulate a whole fibre optic communication system. This surely defines one of the ultimate goals for using SPICE circuit models in simulating the optoelectronic devices and systems.

In conclusion, the SPICE circuit models presented in this work demonstrate the capability and versatility of SPICE in simulating the complicated carrier and lattice heating processes in semiconductor lasers and represent a state of the art modelling tool using leading edge understanding of physical processes. Further enhancement to this work can provide a step towards simulating the optoelectronic devices and systems for fibre optic communication systems.

\section{REFERENCES}

[1] G. F. Carey, W. B. Richardson, C. S. Reed, B, J, Mulvaney, Circuit, Device, and Process Simulation: Mathematical and Numerical Aspects, New York: John Wiley \& Sons, 1996.

[2] P. W. Tuinenga, SPICE: A Guide to Circuit Simulation \& Analysis Using PSpice, 3rd ed.,New Jersey: Prentice-Hall, 1995.

[3] R. S. Tucker, "Circuit model of double-heterojunction laser below threshold," IEE Proc. -Optoeletcron., vol. 128, pp. 101-106, 1981.

[4] R. S. Tucker, "Large-signal circuit model for simulation of injection-laser modulation dynamics," IEE Proc. Optoeletcron., vol. 128, pp. 180-184, 1981.

[5] M. F. Lu, C. Juang, M. J. Jou, B. J. Lee, "Study of carrier transport effects on quantum well lasers using a SPICE simulator," IEE Proc. -Optoeletcron., vol. 142, pp. 237-240, 1995.

[6] M. F. Lu, J. S. Deng, C. Juang, M. J. Jou, and B. J. Lee, “ Equivalent circuit model of quantum-well lasers,” IEEE J. Quantum Electron., vol. 31, pp. 1418-1422, 1995.

[7] N. Bewra, D. A. Suda, G. L. Tan, F. Chatenoud, and J. M. Xu, "Modeling of quantum-well lasers with electro-optothermal interaction," IEEE J. Quantum Electron., vol. 1, pp. 331-340, 1995.

[8] D. S. Ellis and J. M. Xu, "Electro-opto-thermal modeling of threshold current dependence on temperature," IEEE J. Quantum Electron., vol. 3, pp. 640-648, 1997.

[9] Benjamin P. C. Tsou and David L. Pulfrey, "A versatile SPICE model for quantum-well lasers," IEEE J. Quantum Electron., vol. 33, pp. 246-254, 1997.

[10] Benjamin P. C. Tsou and David L. Pulfrey, "The influence of coulomb enhancement on modulation properties of quantum-well lasers," IEEE J. Quantum Electron., vol. 34, pp. 318-324, 1998.

[11] Giammarco Rossi, Roberto Paoletti and Marina Meliga, "SPICE stimulation for analysis and design of fast $1.55 \mu \mathrm{m}$ MQW laser diodes," IEEE J. Lightwave Technol., vol. 16, pp. 1509-1516, 1998.

[12] M. Ganesh Madhan, P. R. Vaya, and N. Gunasekaran, "Circuit Modeling of multimode bistable laser diodes," IEEE Photon. Technol. Lett., vol. 11, pp. 27-29, 1999.

[13] K. Czotscher, S. Weisser, A. Leven and J. Rosenzweig, "Intensity modulation and chirp of 1.55- $\mu \mathrm{m}$ multiplequantum-well laser diodes: modeling and experimental verification," IEEE J. Quantum Electron., vol. 5, pp. 606-612, 1999.

[14] P. V. Mena, J. J. Morikuni, S. M. Kang, A. V. Harton, and K. W. Wyatt, "A simple rate-equation-based thermal VCSEL model," IEEE J. Lightwave Technol., vol. 17, pp. 865-872, 1999.

[15] C. Y Tsai, C. H. Chen, T. L. Sung, C. Y. Tsai, and J. M. Rorison, "Theoretical modeling of carrier and lattice heating effects for frequency chirping in semiconductor lasers," Appl. Phys. Lett., vol. 74, pp. 917-919, 1999.

[16] C. Y. Tsai, C. H. Chen, T. L. Sung, C. Y. Tsai, and J. M. Rorison, "Theoretical modeling of the small-signal modulation response of carrier and lattice temperatures with the dynamics of nonequilibrium optical phonons in semiconductor lasers," IEEE J. Select. Topics Quantum Electron., vol. 5, pp. 596-605, 1999. 